\title{
CARACTERÍSTICAS HEMATOLÓGICAS E PERFIL DE EXPRESSÃO DE ANTÍGENOS MIELÓIDES DE PACIENTES COM LEUCEMIA PROMIELOCÍTICA AGUDA. ANÁLISE DE FATORES PROGNÓSTICOS PARA O DESENVOLVIMENTO DA SÍNDROME DO ÁCIDO RETINÓICO
}

\author{
Flávia Leite Souza Santos, Adriana I. Dore, Ana Sílvia G. Lima, Aglair B. Garcia, \\ Marco A. Zago, Edgar G. Rizzatti, Jorge Elias J', Roberto Passetto Falcão, Eduardo Magalháes Rego* \\ Estudo realizado nas Divisões de Hematologia e de Diagnóstico por Imagem, \\ Departamento de Clínica Médica da Faculdade de Medicina de Ribeirão Preto, SP.
}

RESUMO - OBJETIVO. A leucemia promielocítica aguda (LPA) apresenta uma boa resposta ao tratamento com o ácido all trans retinóico (ATRA). Entretanto, alguns pacientes desenvolvem uma complicação grave chamada síndrome do ácido retinóico (SAR). 0 objetivo deste estudo foi comparar as características hematológicas e imunofenotípicas de pacientes com LPA que desenvolveram a SAR com as daqueles que não a desenvolveram.

MéTODOs. Foram analisados retrospectivamente os prontuá rios, exames radiológicos, lâminas de esfregaço de sangue e medula óssea de 71 pacientes com LPA, dos quais a análise imunofenotípica havia sido realizada em 56 casos. Foram identificados oito casos de SAR que, do ponto de vista clínico, caracterizaram-se por insuficiência respiratória $(n=8)$, insuficiência renal $(n=2)$, febre $(n=5)$, ganho ponderal $(n=3)$, edema periférico $(n=3)$ e derrame pleural $(n=5)$. As seguintes variáveis foram comparadas entre pacientes com e sem SAR: dosagem de hemoglobina, contagens de leucócitos e plaquetas no sangue periférico, distribuição dos subtipos hipergranular e variante, percentagens de blastos $\mathrm{CD}_{3}{ }^{+}, \mathrm{CD} / 3^{+}, \mathrm{CDII7}^{+}$na medula óssea, intensidade e variação dos valores de fluorescência destes antígenos nas células leucêmicas, expressas através dos canais medianos (CMFs) e dos coeficientes de variação (CVs) de fluorescência, respectivamente.

Resultados. A incidência da SAR foi de II,26\% e o tempo médio para seu desenvolvimento II,5 dias do início do tratamento. Todos os pacientes apresentaram desconforto respiratório agudo, por vezes associado à febre, ganho de peso, edema e insuficiência renal. Os achados radiológicos mais comuns foram: opacidades em vidro fosco, derrame pleural, espessamento peribrônquico e aumento da trama vascular pulmonar. Nenhuma das variáveis laboratoriais analisadas correlacionou-se significativamente ao risco de desenvolvimento da SAR, entretanto as Odd Ratios para CMF para o CDII7 > 30 ua e CV para o CD33 < 50 foram de 7,I4 $(P=0,08)$ e de $7,86(P=0,06)$, respectivamente.

Conclusão. A incidência e as características da SAR neste grupo de pacientes brasileiros foi semelhante à descrita na literatura. Nenhum dos parâmetros estudados correlacionou-se significativamente a um maior risco de desenvolvimento desta complicação.

UnITERMOS: Leucemia mielóide aguda. Leucemia promielocítica aguda. Síndrome do ácido retinóico. Imunofenotipagem. Ácido all trans retinóico.

\section{INTRODUÇÃO}

A leucemia promielocítica aguda (LPA) é um subtipo de leucemia mielóide aguda caracterizada pela infiltração da medula óssea por blastos leucêmicos semelhantes a promielócitos'. A LPA corresponde aos subtipos M3 e M3 variante (M3v) de leucemia mielóide aguda segundo a classificação Franco Americo Britânica (FAB), e ao subtipo leucemia mielóide aguda (LMA) associada a translocação entre os cromossomos 15 e $17[t(15 ; \mid 7)]$ e variantes segundo a classificação da Organização Mundial

\footnotetext{
* Correspondência:

Departamento de Clínica Médica Faculdade de Medicina de Ribeirão Preto

Av. Bandeirantes, 3900 - CEP: 14049-900

Ribeirão Preto - SP - Fone: (16) 602-2888

Fax: (16) 633-6695 - E-mail: emrego@hcrp.fmrp.usp.br
}

de Saúde das Neoplasias Mielóides'-3. Do ponto de vista genético, a LPA está sempre associada à translocações cromossômicas envolvendo o locus gênico do Receptor a do Ácido Retinóico (RARa) localizado no braço longo do cromossomo $17^{4}$. Na grande maioria dos casos, a LPA está associada a t $(15 ; \mid 7)$ que causa a fusão entre os genes RARa e Promyelocytic Leukemia (PML), e cria o gene híbrido PMLRARa. Raramente o gene RARa encontra-se fundido aos genespromyelocytic leukemia zinc finger (PLZF), nucleophosmin (NPM), nuclear mitotic apparatus (NuMA) ou signal transducer and activator of transcription 5b(STAT 5b) situados nos cromossomos I I q23, 5q32, I I q I 3 e $|7 q| \mid$, respectivamente ${ }^{5}$. Dada a peculiar resposta da LPA ao tratamento com o ácido all trans retinóico (ATRA), é importante o reco- nhecimento desta forma de leucemia mielóide aguda ${ }^{6}$. O diagnóstico de certeza exige a demonstração das alterações cromossômicas específicas, quer por métodos de citogenética, quer por técnicas de biologia molecular. Entretanto, a citomorfologia e a imunofenotipagem contribuem significativamente para o diagnóstico ${ }^{7-9}$. Orfao et al ${ }^{8}$ demonstraram a existência de um perfil imunofenotípico típico da LPA, caracterizado pela pequena variação nos valores de intensidade de fluorescência do antígeno CD33 (expressão homogênea), que contrasta com a expressão heterogênea do $\mathrm{CD} / 3^{8}$. Ademais, as células leucêmicas são HLA-Dre CD34 negativos, e CDI 5 fracamente positivo ou negativo9,10. O mesmo padrão imunofenotípico foi detectado na variante hipogranular". 
Do ponto de vista clínico, a LPA distingue-se pela elevada freqüência com que se acompanha de coagulação intravascular disseminada e pela resposta ao tratamento ATRA, o qual induz a diferenciação mielóide terminal das células leucêmicas e, associado a agentes antracíclicos, o tratamento com ATRA resulta em taxas de remissão completa superiores a $90 \%$ e de sobrevida livre de doença estimada em três anos superiores a $80 \%{ }^{12-14}$. Estes resultados contrastam com aqueles observados antes da introdução do ATRA no tratamento da LPA, que indicavam sobrevida livre de doença inferiores a 20\% em três anos ${ }^{14}$. Entretanto, o tratamento com ATRA não é isento de complicações, pois de $6 \%$ a $27 \%$ dos pacientes desenvolvem a síndrome do ácido retinóico (SAR), caracterizada por febre de origem inexplicada, ganho de peso, edema generalizado, insuficiência respiratória aguda, derrame pleural e/ou pericárdico, hipotensão arterial e insuficiência renal ${ }^{15-17}$. Os sintomas surgem entre $1^{\circ}$ e $22^{\circ}$ dia do tratamento com ATRA e geralmente (mas não invariavelmente) se associam ao aumento súbito do número de leucócitos no sangue periférico ${ }^{15}$. Seu diagnóstico é clínico, embora anormalidades radiológicas (infiltrado pulmonar em vidro fosco, cardiomegalia, aumento do pedículo vascular pulmonar, linhas septais e derrame pleural) sejam comuns ${ }^{18}$. A análise microscópica dos tecidos afetados mostra a infiltração por células mielóides em diferentes estágios de maturação. Os mecanismos pelos quais - ATRA induz a SAR não são completamente conhecidos e alterações na expressão de moléculas de adesão nos blastos leucêmicos e nas células endoteliais, aumento da permeabilidade capilar, formação de agr egados de leucócitos, e ativação leucocitária induzida por diferentes citocinas já foram implicadas na sua fisiopatologia ${ }^{19-23}$. Ainda que ocorra resposta ao tratamento com dexametasona, a mortalidade associada a SAR é alta (5\%-18\%) e, portanto, é relevante a identificação de pacientes com maior risco de desenvolvê-la $a^{16,17}$.

O presente estudo teve por objetivo analisar parâmetros hematológicos do sangue periférico e da medula óssea, bem como as características imunofenotípicas dos blastos de pacientes com LPA que desenvolveram a SAR e determinar se os mesmos podem estar correlacionados a um maior risco para o desenvolvimento desta complicação.

\section{Métodos}

Foram analisados retrospectivamente os prontuários, exames radiológicos, lâminas de esfregaço de sangue e medula óssea, análises por biologia molecular de 7 I pacientes com LPA diagnosticados e tratados no Hospital das Clínicas de Ribeirão Preto, no período entre 1995 e 2003. Destes, em 56 casos havia também disponível a análise imunofenotípica. 0 diagnóstico de LPA baseou-se nos achados morfológicos nos aspirados de medula óssea, classificados segundo a proposta do grupo $F A B^{\prime}$, e na detecção do transcrito PML-RARa por técnica de reação em cadeia da polimerase (PCR), segundo o descrito por Borrow et a $\left.\right|^{24}$. Todos os pacientes incluídos no presente estudo possuíam a medula óssea infiltrada por mais de $30 \%$ de blastos morfologicamente semeIhantes a promielócitos e foram positivos para a pesquisa do PML-RARa pela reação em cadeia da polimerase segundo o protocolo de Borrow et al ${ }^{24}$. Além disto, os casos foram subclassificados em forma clássica (M3) e variante (M3v), a última caracterizada pela presença de $50 \%$ ou mais dos blastos com granulação citoplasmática pouco evidente ou mesmo ausente e núcleo de contorno irregu$\operatorname{lar}^{9,25}$. Estes blastos foram mieloperoxidase positivos e alfa naftil acetato esterase negativos nas colorações citoquímicas, confirmando sua origem granulocitária ${ }^{26}$.

Os pacientes foram tratados com ATRA associado a quimioterapia baseado no protocolo APL93 do Grupo Europeu de Leucemia Promielocítica Aguda ${ }^{27}$. A indução da remissão consistiu na administração do ATRA via oral na dose de $45 \mathrm{mg} / \mathrm{m}^{2} /$ dia até a remissão completa (CR) ou por no máximo 90 dias. Daunorrubicina $60 \mathrm{mg} / \mathrm{m}^{2} / \mathrm{dia}$ endovenosa (EV) por três dias e AraC $200 \mathrm{mg} / \mathrm{m}^{2} / \mathrm{dia}$ EV por sete dias eram iniciados no $3^{\circ}$ dia do tratamento, independentemente do número de leucócitos. Em raros casos, a introdução da quimioterapia foi postergada até o $70^{\circ}$ dia do tratamento devido à presença de quadro grave de coagulação intravascular disseminada. A consolidação da remissão constitui-se de dois ciclos de quimioterapia sem ATRA, o primeiro dos quais empregou a daunor rubicina e o AraC na mesma posologia descrita para a indução. O segundo ciclo de conso lidação consistia em daunorrubicina $45 \mathrm{mg} /$ $\mathrm{m}^{2} /$ dia EV por três dias e AraC na dose de
I $\mathrm{g} / \mathrm{m}^{2} / \mathrm{dia}$ EV de $12 / \mathrm{I} 2 \mathrm{~h}$ por quatro dias. Todos os pacientes receberam manutenção com ciclos intermitentes de ATRA na dose de $45 \mathrm{mg} / \mathrm{m}^{2} /$ dia durante 15 dias a cada três meses, associados ao uso contínuo de 6 mercaptopurina (90 mg/m²/dia) e meto trexate ( $5 \mathrm{mg} / \mathrm{m}^{2} / \mathrm{semana}$ ) por dois anos. Os critérios de remissão adotados foram: normalização do sangue periférico, presença de menos de $5 \%$ de blastos na medula óssea e negativação do PCR para o PML-RARa.

Nos pacientes com LPA recebendo ATRA, o diagnóstico de SAR foi baseado na presença de pelo menos dois dos seguintes achados sem outras causas detectáveis: desconforto respiratório, febre, ganho de peso, infiltrado pulmonar, derrame pleural ou pericárdico, ou insuficiência renal aguda. Diante do diagnóstico de SAR, a administração do ATRA foi imedia tamente suspensa, e iniciada a terapêutica com dexametasona $10 \mathrm{mg}$ EV de 6/6 horas por três dias. Uma vez tendo ocorrido melhora do quadro clínico, se o paciente tivesse recebido menos de 30 dias de ATRA, a medicação era reintroduzida numa dose $50 \%$ reduzida e lentamente aumentada até a dose preconizada.

Dos 71 pacientes, 56 possuíam análises imunofenotípicas ao diagnóstico. Para esta análise, as células da medula óssea foram incubadas com um painel de anticorpos monoclonais conjugados a ficoeritrina (PE) ou isotiocianato de fluoresceína (FITC) como descrito em Rego et al ${ }^{28}$. Os marcadores estudados foram: CD33, CDI3, CDI I7, HLA-Dr, CDI5, CD34, CD19, CDI0, CD22 e CD3. Nos controles negativos, as amostras foram incubadas com imunoglobulinas de camundongos de especificidade irrelevante, conjugadas aos mesmos fluorocromos. Os anticorpos foram adquiridos da Becton Dickinson já conjugados (Becton Dickinson, Immunocytometry Systems, San Jose, Califórnia, CA). Foi utilizado o citômetro de fluxo FACScan (Becton Dickinson, Immunocytometry Systems, San Jose, Califórnia, CA), cujos procedimentos de calibração e aquisição seguiram rotinas bem estabelecidas. A aquisição e análise de 10.000 a 30.000 células foram feitas empregando-se o programa CellQuest (Becton Dickinson). Para análise selecionou-se a região de blastos/promielocitos definida através dos parâmetros de Forward Scatter Scan (FSC) e Side Scatter Scan (SSC). Foi determinada: a) a percentagem de células positivas 
para os marcadores mielóides CD33, CDI3 e CDII7 na gate; b) a intensidade de fluorescência de cada marcador, analisada pelo canal mediano de fluorescência (CMF) e expressa em unidades arbitrárias (ua) e, c) a variação da fluorescência analisada através do coeficiente de variação $(\mathrm{CV})$ dos canais de fluorescência e expressa em valores contínuos.

A comparação entre os grupos de pacientes que desenvolveram e não desenvolveram a SAR foi feita pelo método não paramétrico usando o Teste $U$ de Mann Whitney para as variáveis contínuas, e pelo Teste Exato de Fisher para as variáveis categóricas. A associação entre variáveis categóricas e o desenvolvimento da SAR foi analisada através das Odds Ratios e o intervalo de confiança 95\% (I.C. 95\%) foi calculado usando a aproximação de Woolf. A hipótese de nulidade foi rejeitada quando a possibilidade de ocorrência casual das diferenças observadas não excedia 5\%. Os cálculos foram efetuados utilizando-se o programa estatístico SPSS para Windows versão 9.0.

\section{Resultados}

Dentre os 71 pacientes com LPA, 6 I (7I,8\%) tinham mais de 15 anos de idade, sendo a mediana da idade de 31 anos e $025^{\circ}$ e $75^{\circ}$ percentis (p25 e p75) iguais a I 5 e 43 anos, respectivamente. A maior freqüência de LPA observada entre pacientes do sexo feminino (42 pacientes; 59, I\% do total) não foi significativamente superior à esperada baseando-se na distribuição por sexo da população brasileira segundo o Censo Demográfico 2000 $\left(50,8 \%\right.$ da população é do sexo feminino, $c^{2}=$ $1,02, P=0,28)$. Oito pacientes $(11,26 \%)$ desenvolveram a SAR, a qual foi diagnosticada entre $\circ 4^{\circ}$ e o $20^{\circ}$ dia (média: II,5 dias) de tratamento com o ATRA. A Tabela I mostra as principais características clínicas destes pacientes. Do ponto de vista clínico, os pacientes com SAR apresentaram desconforto respiratório agudo sem outras causas detectáveis e, em dois casos, houve rápida evolução para insuficiência respiratória necessitando de assistência ventilatória. Nos demais seis pacientes, o uso de $\mathrm{O}_{2}$ nasal ou máscara de Venturi foi suficiente para alivio dos sintomas até a resolução do quadro. Em cinco foi observada febre sem foco infeccioso detectável, e edema periférico e ganho de peso foram detectados em três pacientes. Dois pacientes apresentaram piora importante da função renal (creatinina: 3,I e 3,4 mg/dl; uréia: 84 e $82 \mathrm{mg} / \mathrm{dl}$, respectivamente), necessitando de hemodiálise, porém um destes já era portador de insuficiência renal crônica antes do diagnóstico de LPA e se encontrava em programa ambulatorial de hemodiálise. O desenvolvimento da SAR associou-se ao aumento repentino do número de leucócitos (duplicação em menos de 24 horas) em três pacientes, sendo que em um deles este aumento foi maior que 100 vezes (Tabela I).

O radiograma de tórax revelou opacidades em vidro fosco em todos os casos de SAR, cuja extensão foi variável, e que se acompanharam, na maioria das vezes, de aumento discreto da trama vascular, espessamento peribrônquico e de derrame pleural (Tabela I). Não se detectaram nódulos pulmonares ou linhas septais.

Dois pacientes (25\%) faleceram em decorrência da insuficiência respiratória no $4^{\circ} \mathrm{e}$ $14^{\circ}$ dia de evolução da síndrome. Os demais pacientes apresentaram resolução do quadro pulmonar e a terapêutica com ATRA pôde ser reinstituída de forma paulatina, sendo que a recidiva da SAR foi observada em uma paciente (MA) após I I dias da reintrodução do ATRA, obrigando a suspensão definitiva do mesmo. Os seis pacientes sobreviventes obtiveram remissão completa, hematológica e molecular, com tempo de seguimento variando de 6,4 a 51,6 meses.

A Tabela 2 mostra as contagens hematológicas do sangue periférico e a classificação morfológica quanto aos subtipos clássico e variante ao diagnóstico dos pacientes com LPA. Os pacientes que desenvolveram SAR apresentaram a mediana das contagens de leucócitos mais elevada que aquela detectada em pacientes que não apresentaram a complicação, porém esta diferença não foi significativa $(P=0,27)$. A classificação morfológica nos subtipos clássico e variante foi possível em 63 casos, nos quais a qualidade das preparações de medula óssea foi adequada. Vinte e três dos 63 casos de LPA (36,5\%) foram classificados como subtipo variante, sendo que a freqüência deste subtipo não foi diferente entre os pacientes que apresentaram e aqueles que não apresentaram a SAR $(P=1,0)$ (Tabela 2).

As percentagens de células positivas, os CMFs e os CVs para os marcadores mielóides CDI3, CD33 e CDII7 encontram-se expressas na Tabela 3. Entre todos os 56 pacientes com LPA analisados, observou-se que o CD33 foi o marcador mais intensamente expresso, tendo apresentado pequena variação dos valores de $f$ l u o $\quad r$ e $s$ c ê $n$ c i a (padrão homogêneo). O CDI3 apresentou menor intensidade, mas maior variação da fluorescência (padrão heterogêneo). Comparando o perfil de expressão antigênica entre pacientes que desenvolveram e aqueles que não desenvolveram a SAR, observou-se menor variação da fluorescência do CD33 nos pacientes com a síndrome, porém esta diferença não foi significativa $(P=0,09)$. Da mesma forma, não foram detectadas dife renças significativas nos demais parâmetros de expressão antigênica (Tabela 3).

Com o intuito de identificar fatores de risco para o desenvolvimento da SAR, foram comparadas as distribuições quanto a idade, sexo, parâmetros hematológicos e imunofenotípicos nos pacientes que desenvolveram e nos que não desenvolveram a SAR. Não se observaram diferenças significativas quanto à distribuição etária e por sexo entre os dois grupos (Tabela 4). Para a análise dos parâmetros hematológicos e imunofenotípicos, estes foram categorizados. Os valores cortes (cut off) adotados para a intensidade e variação de fluorescência foram baseados no valor máximo ou mínimo observado nos pacientes que apresentaram a SAR. Não se detectou diferenças significativas quanto à freqüência destas variáveis em pacientes que desenvolveram em comparação àqueles que não desenvolveram a SAR (Tabela 4). Porém, os valores das ORs (I.C. 95\%) para pacientes apresentando CV para o CD33 < 50 e para aqueles com CMF para o CDI I7 > 35 ua foram de 7,86 (0,42 $145,22)$ e de $7,14(0,39$ - 132,23), respectivamente (Tabela 4).

\section{Discussão}

A SAR foi originalmente descrita por Frankel et al. ${ }^{15} \mathrm{em} 1992 \mathrm{em}$ nove de uma série de 35 pacientes com LPA tratados exclusivamente com ATRA. O quadro clínico era muito semelhante ao observado no presente estudo, caracterizado por insuficiência respiratória, febre, ganho ponderal, edema periférico, derrames pleural e/ou pericárdico. Da mesma ma- 


\begin{tabular}{|c|c|c|c|c|c|c|c|c|c|c|}
\hline Paciente & Idade & Sexo & $\begin{array}{l}\text { Insuf. } \\
\text { respiratória }\end{array}$ & $\begin{array}{l}\text { Insuf. } \\
\text { renal }\end{array}$ & Febre & $\begin{array}{l}\text { Opacidade em } \\
\text { vidro fosco }\end{array}$ & $\begin{array}{c}\text { Derrame } \\
\text { pleural }\end{array}$ & $\begin{array}{c}\text { Espessamento } \\
\text { peribrônquico } \\
+\uparrow \\
\text { trama vascular }\end{array}$ & $\begin{array}{l}\text { Contagem de } \\
\text { leucócitos na } \\
\text { véspera do } \\
\text { diagnóstico da } \\
\text { SAR (cels/ } / \text { l) }\end{array}$ & 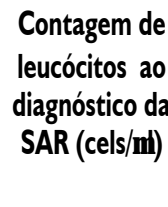 \\
\hline BMD & 38 & $F$ & + & - & + & + & + & + & 600 & 67.100 \\
\hline MA & 41 & $F$ & + & + & + & + & - & - & 700 & 2.100 \\
\hline LGRM & 27 & M & + & - & - & + & - & - & 8.800 & 8.900 \\
\hline F) & 6 & $F$ & + & + & - & + & - & + & 43.100 & 93.300 \\
\hline$C M$ & 24 & $F$ & + & - & + & + & + & + & 800 & 700 \\
\hline OAF & 72 & $F$ & + & - & + & + & + & + & 58.000 & 27.500 \\
\hline VTS & 64 & M & + & - & - & + & + & + & 1.000 & 900 \\
\hline
\end{tabular}

Tabela 2 - Características hematológicas ao diagnóstico dos pacientes com leucemia promielocítica aguda

\begin{tabular}{|c|c|c|c|c|}
\hline & $\begin{array}{c}\text { GB } \\
(\text { cels/ } / \mu l)^{*}\end{array}$ & $\begin{array}{c}\mathrm{Hb} \\
(\mathrm{g} / \mathrm{dl})^{*}\end{array}$ & $\begin{array}{c}\text { PLQ } \\
(\mathrm{plq} / \mu \mathrm{l})^{*}\end{array}$ & Subtipo morfológico \\
\hline Pacientes com LPA $(n=7 \mid)$ & $\begin{array}{c}10.300 \\
(3.350-35.500)\end{array}$ & $\begin{array}{c}8,8 \\
(6.9-10.4)\end{array}$ & $\begin{array}{c}\overline{25.500} \\
(16.000-43.500)\end{array}$ & $23 \mathrm{M3v} / 40 \mathrm{M3}$ \\
\hline $\begin{array}{l}\text { Pacientes com LPA que desenvolveram a SAR } \\
(n=8)\end{array}$ & $\begin{array}{c}29.800 \\
(3.500-85.925)\end{array}$ & $\begin{array}{c}10,2 \\
(6,8-11,1)\end{array}$ & $\begin{array}{c}30.000 \\
(21.250-50.000)\end{array}$ & 3 M3v / 5 M3 \\
\hline $\begin{array}{l}\text { Pacientes com LPA que não desenvolveram a SAR } \\
(\mathrm{n}=63)\end{array}$ & $\begin{array}{c}9.000 \\
(3.400-33.700)\end{array}$ & $\begin{array}{c}8,5 \\
(7,1-9,9)\end{array}$ & $\begin{array}{c}26.000 \\
(15.000-49.000)\end{array}$ & $20 \mathrm{M3v} / 35 \mathrm{M3}$ \\
\hline
\end{tabular}

"Valores representam a mediana $\left(25^{\circ}-75^{\circ}\right.$ percentis). ${ }^{* "}$ Calculado pelo Teste de Mann-Whitney, ${ }^{* " *}$ Calculado pelo Teste Exato de Fisher.

Tabela 3 - Características imunofenotípicas das células leucêmicas de pacientes com leucemia promielocítica aguda

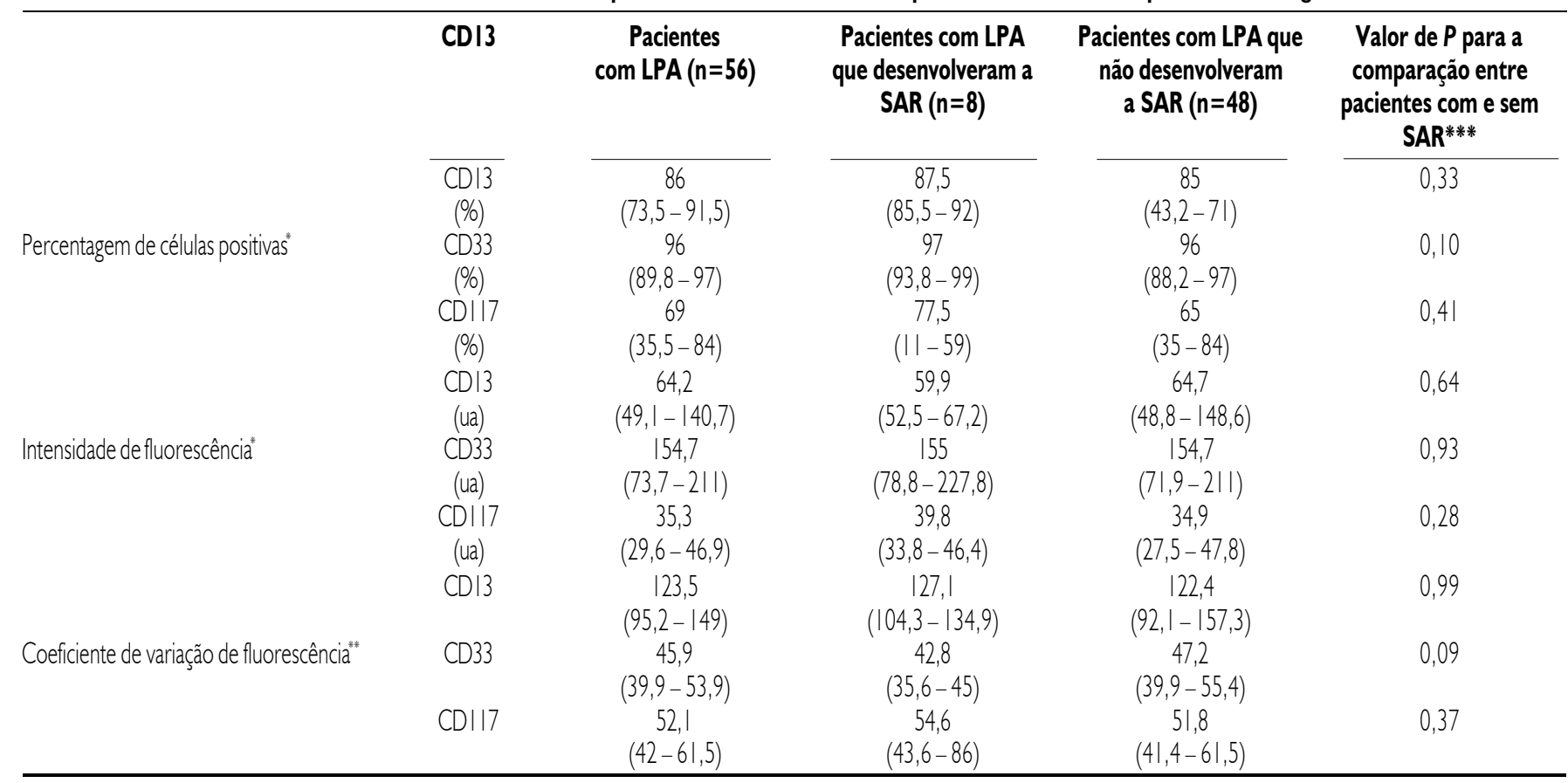

Valores representam a mediana (250 - $75^{\circ}$ percentis). * Expresso pelo Canal Mediano de Fluorescência, em unidades arbitrárias (ua); ** Expresso pela Mediana do Coeficiente de Variação dos Canais de Fluorescência.

"*alculado pelo Teste U de Mann-Whitney. 


\section{Tabela 4 - Análise da distribuição das características clínicas e laboratoriais entre pacientes com leucemia promielocítica aguda que desenvolveram ou não a síndrome do ácido retinóico}

\begin{tabular}{|c|c|c|c|c|}
\hline Variável & $\begin{array}{c}\text { Freqüência nos pacientes } \\
\text { com LPA que desenvolveram } \\
\text { a SAR }(n=8)\end{array}$ & $\begin{array}{c}\text { Freqüência nos pacientes } \\
\text { com LPA que não } \\
\text { desenvolveram a SAR }(n=63)\end{array}$ & Odds Ratio** & Valor de $P * * *$ \\
\hline Idade & 32,5 anos $(10,5-58,2)^{*}$ & 31 anos $(15-43)^{*}$ & n.a. & 0,8 \\
\hline Hemoglobina $\leq \log / \mathrm{dl}$ & $4 / 8(50 \%)$ & $49 / 63(77,8 \%)$ & $0,28(0,06-1,29)$ & 0,11 \\
\hline Leucócitos $\geq 5.000 / \mu \mid$ & $6 / 8(75 \%)$ & $39 / 63(61,9 \%)$ & $1,85(0,34-9,9)$ & 0,38 \\
\hline Intensidade CD33 > 55 ua & $8 / 8(100 \%)$ & $42 / 48(87,5 \%)$ & $2,6(0,13-50,68)$ & 0,38 \\
\hline Intensidade CDI3 < 360 ua & $8 / 8(100 \%)$ & $42 / 48(87,5 \%)$ & $2,6(0,13-50,68)$ & 0,38 \\
\hline Intensidade CDI |7 > 30 ua & $8 / 8(100 \%)$ & $34 / 48(70,8 \%)$ & $7,14(0,39-132,23)$ & 0,08 \\
\hline C.V. CD33 $<50$ & $8 / 8(100 \%)$ & $33 / 48(68,7 \%)$ & $7,86(0,42-145,22)$ & 0,06 \\
\hline C.V. CDI3 $<200$ & $8 / 8(100 \%)$ & $40 / 48(83,3 \%)$ & $3,57(0,19-67,98)$ & 0,27 \\
\hline C.V. CDII7 > 35 & $8 / 8(100 \%)$ & $43 / 48(89,6 \%)$ & $2,15(0,11-42,64)$ & 0,45 \\
\hline
\end{tabular}

* Valores representam a mediana (percentis $25^{\circ}-75^{\circ}$ ). *** Valores representam Odds Ratio (Intervalo de confiança $95 \%$ ). O intervalo de confiança $95 \%$ foi calculado usando a aproximação de Woolf .*** Calculado pelo Teste Exato de Fisher.

neira, os achados radiológicos descritos aqui também foram semelhantes aos descritos por Jung et al. ${ }^{18}$ e não são específicos da SAR. A incidência da SAR na literatura variou entre $6 \%$ a $27 \%$ 15-17,19,29, refletindo as diferenças quanto à associação ou não de antracíclicos e quanto ao momento de sua introdução. No presente estudo, a incidência da SAR foi de $11,26 \%$, semelhante à descrita por De Botton et al. 17,30 usando o mesmo protocolo de tratamento. A importância da introdução do antracíclico nos primeiros dias do tratamento com ATRA na prevenção da SAR foi estudada pelo Grupo Europeu para o Estudo da LPA, o qual encontrou uma incidência da SAR de $18 \%$ no grupo de pacientes que recebeu quimioterapia após o uso prolongado de ATRA (até a obtenção de remissão), em contraste com uma incidência de apenas 9,2\% no grupo que recebeu a mesma a partir do $3^{\circ}$ dia do tratamento ${ }^{30}$. Ademais, a mortalidade por SAR foi menor no segundo grupo (0,5\% versus 2,5\%). Em relação à mortalidade, esta variou entre $0,5 \%$ e $29 \%$, embora estudos mais recentes tenham observado índices inferiores a 10\% 16,19,30,31. Assim, a mortalidade observada no presente estudo (25\%) foi elevada, o que não parece decorrer do tratamento usado, uma vez que o mesmo (administração de dexametasona em altas doses) foi semelhante àquele adotado por Frankel et al. ${ }^{15}$ e por Vahdat et al..$^{19}$ que descreveram percentagens de óbito em pacientes com SAR de $4,5 \%$ e de $5,25 \%$, respectiva- mente. Deve-se levar em conta o tamanho de nossa amostra e o fato de um dos pacientes falecidos da SAR apresentar insuficiência renal crônica anterior ao diagnóstico da LPA.

Estudos anteriores analisaram a correlação entre idade, sexo, as contagens do sangue periférico ao diagnóstico, o subtipo morfológico e a isoforma do transcrito PML-RARa com o desenvolvimento da SAR ${ }^{16,17,19}$. Nosso estudo corrobora os anteriores, nos quais não se detectou maior freqüência da SAR em um grupo etário ou em um dos sexos ${ }^{16,17,19}$. A semelhança do trabalho de De Botton et al. ${ }^{17}$ também não detectou diferenças significativas quanto às contagens de leucócitos ao diagnóstico entre pacientes que desenvolveram ou não a SAR. Estes dados contrastam com o estudo de Fenaux et al. ${ }^{32}$ que descreveram que 0 número de leucócitos ao diagnóstico correlacionou-se ao desenvolvimento da SAR. Porém, esta diferença possivelmente reflete a disparidade quanto ao tempo para a introdução do antracíclico. É interessante notar que no estudo de Vahdat et al. ${ }^{19}$ também não houve correlação entre o número de leucócitos ao diagnóstico e o desenvolvimento da SAR mas, ao contrário, o valor máximo deste número após a introdução do ATRA apresentou importante associação.

Tallman et al. ${ }^{16}$ afirmaram que pacientes com o subtipo morfológico variante estariam protegidos da SAR. Entretanto, estes achados não foram confirmados em estudos com maior casuística ${ }^{17}$ e é provável que esta discrepância reflita as diferenças na classificação morfológica. De fato, a freqüência do subtipo variante na literatura varia de $15 \%$ a $38 \%{ }^{33-35}$. No presente estudo, a freqüência deste subtipo foi de $36,5 \%$, e não se observou diferença significativa na freqüência da SAR entre pacientes portadores do subtipo morfológico clássico e variante da LPA.

A imunofenotipagem tem um papel importante no diagnóstico da LPA. O estudo multiparamétrico, em particular, é capaz de distinguir a vasta maioria dos casos de LPA dos demais subtipos de LMA, como o demonstrado por Orfao et al ${ }^{8}$. Os estudos anteriores, analisando a associação do imunofenótipo com o desenvolvimento da SAR, basearam-se na análise qualitativa da expressão (positividade ou negatividade para um dado marcador). Assim, Vahdat et al. ${ }^{19}$ encontraram que a expressão basal do CDI 3 nos blastos da LPA estava associada ao desenvolvimento da SAR. O presente estudo foi o primeiro a analisar a associação entre diferenças quantitativas e qualitativas na expressão de antígenos mielóides e o desenvolvimento da SAR. A intensidade de fluorescência, aqui estudada através do CMF, é diretamente proporcional à densidade antigênica como o demonstrado por Poncelet \& Carayon ${ }^{36}$. Ela pode ser mais precisamente analisada através da técnica de quantimetria, na qual esferas com quantidades conhecidas de antígenos são usadas para criar uma curva de 
calibração relacionando CMF e densidade antigênica $^{37}$. Porém, considerando os protocolos atuais para a calibração do citômetro, o uso diretamente do CMF é um método adequado e reprodutível. Em relação à variação da intensidade de fluorescência, Orfao et al. ${ }^{8}$ demonstraram que a expressão do CDI3 na LPA apresenta grande variação (acima de uma dezena logarítmica da escala de intensidade de fluorescência), ao que chamaram de padrão heterogêneo, contrastando com o padrão obser-vado para o CD33. No presente estudo, o mesmo perfil de expressão do CDI3 e CD33 foi encontrado, porém, o aspecto mais relevante foi a demonstração da associação entre valores de CV para o CD33 < 50 e de CMF para o CDII7 > 30 ua com um maior risco de desenvolvimento da SAR. Estas diferenças tiveram significância estatística marginal, provavelmente em virtude do reduzido número de casos analisados. Porém, considerando a baixa incidência da SAR e sua gravidade clínica, os resultados apresentados aqui são relevantes e justificam estudos multicêntricos futuros analisando o perfil de expressão do CD33 e do CDII7 em uma maior coorte de pacientes.

\section{Conclusões}

O presente estudo mostra que a freqüência e as características clínicas, radiológicas e laboratoriais da SAR neste grupo de pacientes brasileiros foi semelhante à descrita na literatura. Nenhum parâmetro clínico e laboratorial foi capaz de identificar quais os pacientes com LPA que irão desenvolver esta complicação.

Conflito de interesse: não há.

\section{SUMMARY}

\section{Hematological features and expres- SION PROFILE OF MYELOID ANTIGENS OF ACUTE PROMYELOCYTIC LEUKEMIA PATIENTS. ANAL- YSIS OF PROGNOSTIC FACTORS FOR DEVELO- PMENT OF THE RETINOIC ACID SYNDROME}

BACKGROUND. Acute Promyelocytic Leukemia (APL) is characterized by its good response to treatment with all trans retinoic acid (ATRA). However, some patients receiving ATRA develop a serious complication called retinoid syndrome (RS). The objective of this study was to compare the hematological and immunophenotypic features of APL patients who developed RS with those who did not.

METHODS. We reviewed the medical records, roentgenograms, peripheral blood smears and bone marrow aspirates from 7I APL patients. Immunophenotypic analyses were available in 56 of these cases. Eight cases of RS were detected, whose clinical presentation was characterized by respiratory distress $(n=8)$, impairment of the renal function $(n=2)$, fever $(n=5)$, weight gain $(n=3)$, edema $(n=3)$ and/or pleural effusion $(n=5)$. The following variables were compared in patients with and without RS: hemoglobin levels, leukocyte and platelet counts, frequency of hypergranular and variant morphological subtypes, percentages of $\mathrm{CD}_{3}{ }^{+}, \mathrm{CDI}^{+}$, $\mathrm{CDI} 17^{+}$blasts in the bone marrow, fluorescence intensity and variation of these markers in the leukemic cells, expressed as the median channel of fluorescence (MCF) and fluorescence coefficient of variation (CV), respectively.

RESULTS. RS incidence was $11.26 \%$ and the average time for syndrome development was I I. 5 days after starting ATRA treatment. All patients presented acute respiratory distress. Other symptoms included fever, weight gain, edema and renal insufficiency. The main radiological findings were ground glass opacities, increased vascular pedicle and peribronchial cuffing. There was no significant correlation between the variables selected and the risk of development of RS, however the Odds Ratios for patients presenting MCF for CDII $7>30$ ua and CV for $\mathrm{CD} 33<50$ were of $7.14(P=0.08)$ and $7.86(P=0.06)$, respectively.

Conclusions. The incidence, as well as the clinical, radiological and laboratory features of RS in this group of Brazilian APL patients were similar to those described in literature. None of the variables studied were significantly correlated to a higher risk of developing RS. [Rev Assoc Med Bras 2004; 50(3): 286-92]

KEY wORDs: Acute myeloid leukemia. Acute Promyelocytic leukemia. Retinoid syndrome, All trans retinoic acid. Immunophenotyping.

\section{RefERÊNCIAS}

I. Bennett JM, Catovsky D, Daniel MT, Flandrin G, Galton DA, Gralnick HR, et al. Proposals for the classification of the acute leukaemias. French- American-British (FAB) co-operative group. Br J Haematol 1976; 33:45 I-8.

2. Vardiman JW, Harris NL, Brunning RD. The
World Health Organization (WHO) classification of the myeloid neoplasms. Blood 2002; 100:2292-302.

3. Brunning RD, Matutes E, Flandrin G, Vardiman J, Bennett J, Head D, et al. Acute myeloid leukaemia with recurrent genetic abnormalities. In: Jaffe ES, Harris NL, Stein H, Vardiman JW, editors. Pathology and genetics of tumours of haematopoietic and lymphoid tissues. Lyon: IARC Press; 200I. p. 8I-7.

4. Rego EM, Pandolfi PP. Analysis of the molecular genetics of acute promyelocytic leukemia in mouse models. Semin Hematol 2001; 38:54-70.

5. Melnick A, Licht JD. Deconstructing a disease: RARalpha, its fusion partners, and their roles in the pathogenesis of acute promyelocytic leukemia. Blood 1999; 93:3167-215.

6. Warrell RP Jr., De The H, Wang ZY, Degos L. Acute promyelocytic leukemia. N Engl J Med 1993; 329: 177-89.

7. Sainty D, Liso V, Cantu-Rajnoldi A, Head D, Mozziconacci MJ, Arnoulet C, et al. A new morphologic classification system for acute promyelocytic leukemia distinguishes cases with underlying PLZF/RARA gene rearrangements. Group Francais de Cytogenetique Hematologique, UK Cancer Cytogenetics Group and BIOMED I European CoomunityConcerted Acion "Molecular Cytogenetic Diagnosis in Haematological Malignancies. Blood 2000; 96: I 287-96.

8. Orfao A, Chillon MC, Bortoluci AM, LopezBerges MC, Garcia-Sanz R, Gonzalez M, et al. The flow cytometric pattern of CD34, CDI 5 and $\mathrm{CDI} 3$ expression in acute myeloblastic leukemia is highly characteristic of the presence of PML-RARalpha gene rearrangements. Haematologica 1999; 84:405- 12.

9. Neame PB, Soamboonsrup P, Leber B, Carter RF, Sunisloe L, Patterson W, et al. Morphology of acute promyelocytic leukemia with cytogenetic or molecular evidence for the diagnosis: characterization of additional microgranularvariants. Am J Hematol I 997; 56: I 3 | -42.

10. Orfao A, Ciudad J, Gonzalez M, Lopez A, Del Mar Abad M, Paz Bouza Jl, et al. Flow cytometry in the diagnosis of cancer. Scand J Clin Lab Invest Suppl 1995; 22 1: | 45-52.

II. Rizzatti EG, Portieres FL, Martins SLR, Rego EM, Zago MA, Falcão RP. The microgranular and the $t(11 ; 17) / P L Z F-R A R a$ variants of acute promyelocytic leukemia also present the flow cytometric pattern of CD I3, CD34 and CD I 5 expression characteristic of PML-RARa gene rearrangement. Am J Hematol 2004;76:44-5I.

12. Fenaux P, Chevret $S$, Guerci A, Fegueux N, Dombret $\mathrm{H}$, Thomas $X$, et al. Long-term followup confirms the benefit of all-trans retinoic acid in acute promyelocytic leukemia. European APL group. Leukemia 2000; 14: 1371-7.

13. Fenaux P, Chomienne C, Degos L. Treatment of acute promyelocytic leukaemia. Best Pract Res Clin Haematol 200 I ; 4: I 53-74.

14. Fenaux P, Chomienne C, Degos L. All-trans retinoic acid and chemotherapy in the treatment of acute promyelocytic leukemia. Semin 
Hematol 200I; 38:13-25.

15. Frankel SR, Eardley A, Lauwers G, Weiss M, Warrell RP Jr. The "retinoic acid syndrome" in acute promyelocytic leukemia. Ann Intern Med 1992; 117:292-6.

16. Tallman MS, Andersen JW, Schiffer CA, Appelbaum FR, Feusner JH, Ogden A, et al. Clinical description of 44 patients with acute promyelocytic leukemia who developed the retinoic acid syndrome. Blood 2000; 95:90-5.

17. De Botton S, Dombret H, Sanz M, Miguel JS, Caillot D, Zittoun R, et al. Incidence, clinical features, and outcome of all trans-retinoic acid syndrome in 413 cases of newly diagnosed acute promyelocytic leukemia. The European APL Group. Blood 1998; 92:2712-8.

18. Jung JI, Choi JE, Hahn ST, Min CK, Kim CC, Park SH. Radiologic features of all-transretinoic acid syndrome. AJR Am J Roentgenol 2002; 178:475-80

19. Vahdat L, Maslak P, Miller WH, Jr., Eardley A, Heller G, Scheinberg DA, et al. Early mortality and the retinoic acid syndrome in acute promyelocytic leukemia: impact of leukocytosis, low-dose chemotherapy, PMN/RARa isoform, and CDI3 expression in patients treated with all-trans retinoic acid. Blood 1994; 84:3843-9.

20. Di Noto R, Schiavone EM, Ferrara F, Manzo C, Lo Pardo C, Del Vecchio L. Expression and ATRA-driven modulation of adhesion molecules in acute promyelocytic leukemia. Leukemia 1994; 8: 1900-5.

21. Seale J, Delva L, Renesto P, Balitrand N, Dombret $\mathrm{H}$, Scrobohaci $\mathrm{ML}$, et al. All-trans retinoic acid rapidly decreases cathepsin $G$ synthesis and mRNA expression in acute promyelocytic leukemia. Leukemia 1996; 10:95-101.

22. Dubois C, Schlageter MH, De Gentile A, Balitrand N, Toubert ME, Krawice I, et al. Modulation of IL-8, IL-I beta, and G-CSF secretion by all-trans retinoic acid in acute promyelocytic leukemia. Leukemia 1994;
8:1750-7.

23. Larson RS, Brown DC, Sklar LA. Retinoic acid induces aggregation of the acute promyelocytic leukemia cell line NB-4 by utilization of LFA-I and ICAM-2. Blood I997; 90:2747-56.

24. Borrow J, Goddard AD, Gibbons B, Katz F, Swirsky D, Fioretos T, et al. Diagnosis of acute promyelocytic leukaemia by RT-PCR: detection of PML-RARA and RARA-PML fusion transcripts. Br J Haematol 1992; 82:529-40.

25. Bennett JM, Catovsky D, Daniel MT, Flandrin G, Galton DA, Gralnick HR, et al. A variant form of hypergranular promyelocytic leukaemia (M3). Br J Haematol 1980; 44: I 69-70.

26. Li C-Y, Yam LT, Sun T. Modern modalities for diagnosis of hematologic neoplasms. New York: Igaku-Shoin; 1996.

27. Fenaux $P$, Chastang $C$, Chevret $S$, Sanz $M$, Dombret $\mathrm{H}$, Archimbaud $\mathrm{E}$, et al. A randomized comparison of all transretinoic acid (ATRA) followed by chemotherapy and ATRA plus chemotherapy and the role of maintenance therapy in newly diagnosed acute promyelocytic leukemia. The European APL Group. Blood 1999; 94: I 192-200.

28. Rego EM, Tone LG, Garcia AB, Falcao RP. CDIO and CDI9 fluorescence intensity of B-cell precursors in normal and leukemic bone marrow. Clinical characterization of CDIO (+strong) and CDIO(+weak) common acute lymphoblastic leukemia. Leuk Res 1999: 23:44|-50.

29. Kanamaru A, Takemoto $Y$, Tanimoto $M$, Murakami H, Asou N, Kobayashi T, et al. Alltrans retinoic acid for the treatment of newly diagnosed acute promyelocytic leukemia. Japan Adult Leukemia Study Group. Blood 1995; 85: | 202-6.

30. De Botton S, Chevret S, Coiteux V, Dombret H, Sanz M, San Miguel J, et al. Early onset of chemotherapy can reduce the incidence of ATRA syndrome in newly diagnosed acute promyelocytic leukemia (APL) with low white blood cell counts: results from APL 93 trial. Leukemia 2003; 17:339-42.

31. Avvisati G, Lo Coco F, Diverio D, Falda M, Ferrara F, Lazzarino M, et al. AIDA (alltrans retinoic acid + idarubicin) in newly diagnosed acute promyelocytic leukemia: a Gruppo Italiano Malattie Ematologiche Maligne dell' Adulto (GIMEMA) pilot study. Blood 1996; 88:1390-8.

32. Fenaux $P$, Castaigne $S$, Chomienne C, Dombret $H$, Degos L. All trans retinoic acid treatment for patients with acute promyelocytic leukemia. Leukemia 1992; 6:64-6.

33. Avvisati G, Petti MC, Lo-Coco F, Vegna ML, Amadori S, Baccarani $M$, et al. Induction therapy with idarubicin alone significantly influences event-free survival duration in patients with newly diagnosed hypergranular acute promyelocytic leukemia: final results of the GIMEMA randomized study LAP 0389 with 7 years of minimal follow-up. Blood 2002; 100:3141-6.

34. Avvisati G, Lo Coco F, Mandelli F. Acute promyelocytic leukemia: clinical and morphologic features and prognostic factors. Semin Hematol 200 I; 38:4-12

35. Guglielmi C, Martelli MP, Diverio D, Fenu S, Vegna ML, Cantu-Rajnoldi A, et al. Immunophenotype of adult and childhood acute promyelocytic leukaemia: correlation with morphology, type of PML gene breakpoint and clinical outcome. A cooperative Italian study on 196 cases. Br J Haematol 1998; 102: |035-4|.

36. Poncelet P, Carayon P. Cytofluorometric quantification of cell-surface antigens by indirect immunofluorescence using monoclonal antibodies. J Immunol Methods 1985: 85:65-74.

37. Rego EM, Garcia AB, Carneiro JJ, Falcao RP. Immunophenotype of normal and leukemic bone marrow B-precursors in a Brazilian population. A comparative analysis by quantitative fluorescence cytometry. Braz I Med Biol Res 2001; 34: 183-94.

Artigo recebido: 30/07/2003

Aceito para publicação: 16/12/2003 